\title{
The Explore of Third Molar for Chronological Age Estimation in Teenagers of Shanghai Han Population
}

\section{Ge-fei Shi ${ }^{1}$, Xiao-peng $\mathrm{Li}^{2}$, Rui-jue Liu ${ }^{1}$, Jiang Tao ${ }^{3}$ and Guang-you Zhu ${ }^{1 \star}$}

${ }^{1}$ Shanghai Key Laboratory of Forensic Medicine, Institute of Forensic Science, Ministry of Justice, PR China

2Shanghai Shenyuan Yixue Forensic Science Institution, PR China

${ }^{3}$ Shanghai Ninth People's Hospital Affiliated to School of Medicine, Shanghai Jiao Tong University, PR China

"Corresponding author: Guang-you Zhu, Shanghai Key Laboratory of Forensic Medicine, Institute of Forensic Science, Ministry of Justice, PR China, Tel: 8602162442691; E-mail: zhugy@ssfj.cn

Received date: November 01, 2014; Accepted date: January 09, 2015; Published date: January 15, 2015

Copyright: $\odot 2018$ Shi G, et al. This is an open-access article distributed under the terms of the Creative Commons Attribution License, which permits unrestricted use, distribution, and reproduction in any medium, provided the original author and source are credited.

\begin{abstract}
Objective: To study the applicability of third molar for age estimation in teenager of Shanghai Han population in China.

Methods: The third molars of 501 Han population teenagers of Shanghai from Shanghai Ninth Hospital were classified with Demirjian's classification method. There were 168 males and 333 females involved in this study and the chronological ages ranged from 11 to 20 years. Wilcoxon test and correlation analysis was performed to find the difference between third molars. Mann-Whitney and T-test were employed to find the difference between the males and females.

Results: (1) The results of Mann-Whitney and T-test showed that there is no difference in the growth of third molars between males and females. (2) Pearson test illustrated that there was a high correlation between 18 and 28 , 38 and 48 (girl $R=0.90$ and 0.92 , boy $R=0.91$ and $0.92, P<0.01$ ), while there was a low correlation between 18 and 48,28 and 38 (girl $R=0.57$ and 0.51 , boy $R=0.53$ and $057, P<0.01$ ). Wilcoxon test showed that there is no statistical significance between two side molars $(P>0.05)$, on the contrast, outstanding statistical significance between mandibular and maxillary molars $(P<0.01)$. (3) When the classification of wisdom molar reached to $C, D$ and $G$, more than $83 \%$ is under 14 -year, more than $97 \%$ under 16 -year, more than $57 \%$ over 16 -year and more than $50 \%$ under 18 -year respectively. Conclusion Third molar could be used to estimate the age of teenagers. But there is different accuracy rate for the different classification of the third molar.
\end{abstract}

Keywords: Forensic dentistry; Age determination; The third molar; Demirjian's method; Adolescent; Han nationality

\section{Introduction}

It is an important and difficult job to determine chronological age. The latest Meta-Analysis find that Demirjian's classification method could be used to estimate 3.5 16.9 years old people by permanent teeth except third molar [1]. It is well known that third molar erupt after 18-year-old and grow completely after 22-year-old. So, they are used to estimate over 16-year-old teenagers, though the development of third molar is different from people to people. However, it is an effective method to estimate age.

Olze et al. [2] found that there is difference among different races assessing age by third molar with Demirjian's classification method. And there is no study data in the field in China. Based on the reliability and operability of Demirjian's classification method, our research is to find out the degree of mineralization of third molar in young people and to estimate the chronological age.

\section{Materials and Methods}

\section{Materials}

In this retrospective study, orthopantomograms of 501 Chinese Han people of known chronological age and gender were selected; 168 were males and 333 were females and their ages ranged from 11 to 20 years. The radiographs of healthy children were randomly selected from patients attending the Orthodontic Department of Shanghai Ninth People's Hospital Affiliated to Shanghai Jiao Tong University School of Medicine, Shanghai. Table 1 shows the gender and age distribution of orthopantomograms. Table 2 shows the gender distribution of third molar. The criteria for inclusion in the sample were the availability in their clinical records of an orthopantomogram of adequate quality, and no history of medical or surgical disease that could affect the presence and development of mandibular permanent teeth. Exclusion criteria included: image deformity affecting mandibular permanent tooth visualization, hypodontia, or gross pathology. The chronological age for each subject was calculated by the date of taking panoramic radiograph and the date of birth, having been converted to a two decimal age. 
Page 2 of 7

\section{Methods}

Every third molar was classified by two experts according to the Demirjian's classification method. When the development of a tooth is between two stages, its stage was identified to the previous stage in our study. And this rule is also used in the situation when two experts gave different grades.

In the study, Federation Dentaire International Numbering System (FDI) is employed. The maxillary right third molar is marked as 18 , the maxillary left third molar is marked as 28 , the mandibular left third molar is marked as 38 , and the mandibular right third molar is marked as 48 .

Microsoft $^{\star}$ Office $^{E^{2}}{ }^{\triangleright} 2007$ was employed to manage data, which include chronological age, gender, stages of mineralization of each third molar and so on.

\begin{tabular}{|l|l|l|l|l|}
\hline Age & Male & Female & Summary & Frequent (\%) \\
\hline $11.00 \sim$ & 14 & 36 & 50 & 9.98 \\
\hline $12.00 \sim$ & 37 & 89 & 126 & 25.15 \\
\hline $13.00 \sim$ & 47 & 90 & 137 & 27.35 \\
\hline $14.00 \sim$ & 33 & 46 & 79 & 15.77 \\
\hline $15.00 \sim$ & 22 & 29 & 51 & 10.18 \\
\hline $16.00 \sim$ & 10 & 20 & 30 & 5.99 \\
\hline $17.00 \sim$ & 4 & 10 & 14 & 2.79 \\
\hline $18.00 \sim$ & 1 & 7 & 8 & 1.60 \\
\hline $19.00 \sim 20.00$ & 0 & 6 & 6 & 1.20 \\
\hline Summary & 168 & 333 & 501 & 100.00 \\
\hline
\end{tabular}

Table 1: Age and sex distribution (Han).

\begin{tabular}{|l|l|l|l|}
\hline Age & Male & Female & Summary \\
\hline 18 & 135 & 257 & 392 \\
\hline 28 & 144 & 252 & 396 \\
\hline 38 & 156 & 298 & 454 \\
\hline 48 & 155 & 298 & 453 \\
\hline Summary & 590 & 1105 & 1695 \\
\hline
\end{tabular}

Table 2: Sex distribution of every third molar.

\section{Statistics analysis}

In our study, Wilcoxon and Pearson test was performed to find out the correlation between any two third molars. Meanwhile, the difference of development of third molar in gender was tested by Mann-Whitney U test.

For every classification of third molar, mean age \pm standard deviation, $\max , \min$ and percentile $(25 \%, 50 \%$ and $70 \%)$ were calculated. After this, the further tests were done, which include independent $\mathrm{T}$ test and the accuracy of assessing 14, 16 and 18 respectively.
All of statistics analysis was performed by SPSS15. $0^{\circ}$ for Windows.

\section{Results}

The results of Pearson test and Mann-Whitney U test are listed in Tables 3-5. These illustrates that there is strong correlation between 18 and 28, 38 and 48 (girl, $\mathrm{R}=0.90$ and 0.92 , boy, $\mathrm{R}=0.91$ and 0.92 , $\mathrm{P}<0.01$ ), while there is weak correlation between 18 and 48, 28 and 38 (girl, $\mathrm{R}=0.57$ and 0.51 , boy, $\mathrm{R}=0.53$ and $0.57, \mathrm{P}<0.01$ ). Wilcoxon test (Table 5 shows that there is no statistical significance between 18 and 28,38 and $48(\mathrm{P}>0.05)$ and notable statistical significance between 18 and 48,28 and $38(\mathrm{P}<0.01)$.

The mean age \pm standard deviation, $\max , \min$ and percentile $(25 \%$, $50 \%$ and $70 \%$ ) of every classification of third molar is showed in Tables 6 (male) and 7 (female) respectively. T-test proved that there is no statistical significance between boy and girl $(\mathrm{P}>0.05)$. Tables 8 and 9 shows the probability to estimate legal sensitive age 14-year old age, 16-year old age and 18-year old age.

\section{Discussion}

The accuracy of age determined by tooth is various by different methods. Those who are older than 12 years age have got 28 permanent teeth expect the third molar, which always erupt in 18-year and completely calcify after 30-year. Sometimes, third molar never erupt in the life. However, third molar can be used to determine the youngster's age.

Olze et al. [3] found more reliable with Demirjian's method than Gleiser and Hunt method, Gustafson and Koch method, Harris and Nortje method, and Kullman method. Besides above, the great reason selected by our study contributed to the highest relationship between determined age and chronological age.

It is reported by several studies that linear correlation between the development of third molar and chronological age is marked [4-7]. While our study shows that the relationship is higher in female than in male, the contrary result ( $\mathrm{r} 2$ in male $=0.54, \mathrm{r} 2$ in female $=0.45$ ) is found by Prieto et al. [8].

There is high relationship (Table 5) between 18 and 28, 38 and 48 respectively, certified by other research $[4,8-14]$. There is, however, no relationship between 18 and 48,28 and 38 . And the result is proved by Wilcoxon test (Table 5). In female, when the third molar is classified as stage $\mathrm{A}, \mathrm{B}, \mathrm{C}, \mathrm{F}, \mathrm{G}$ and $\mathrm{H}$, the chronological age estimated by 18 is underestimated 0.72-year age, 0.10-year age, 0.09-year age, 2.64-year age, 0.15 -year age and 0.68 -year age respectively than by 48 . Meanwhile, 0.40-year age, 0.06-year age, 0.24-year age, 0.52-year age, 1.42 -year age and 0.23 -year age is underestimated by 28 respectively than by 38 . When the third molar is classified as stage $D$, the chronological age estimated by 18 and 28 is overestimated to 0.54 -year age and 0.52 -year age respectively than by 48 and 38. It's a pity that the 18 and 28 subjects in stage $\mathrm{E}$ of both female and male, in stage $\mathrm{F}$ and $\mathrm{A}$ of male is absence. In male, when the third molar is classified as B and $\mathrm{G}$, the chronological age is underestimated to be 0.20 -year age and 0.81 -year age by 18 than 48 and 0.37 -year age and 0.81 -year age by 28 than 38. When the stage of classification is C, D and H, 0.15-year age, 0.37 -year age and 2.06-year age are overestimated by 18 than 48 and 0.26 -year age, 0.45 -year age and 2.06-year age by 28 than 38 .

As a consequent, our study shows that the mineralization of maxillary third molar is earlier, faster than mandibular one in female. 
Citation: Ge-fei Shi, Xiao-peng Li, Rui-jue Liu, Jiang Tao3, Guang-you Zhu (2014) The Explore of Third Molar for Chronological Age Estimation

Page 3 of 7

Those are in common with the results of Olze et al. [15] and Bolanos et al. [16].

The mineralization difference of third molar between male and female is not found by Mann-Whitney test and T-test (Tables 4 and 5). And this is in common with the results studied by Orhan et al. [4] in Turkey and by Olze et al. [17] in Japan. The male third molar mineralizes earlier than female in west south China [14]. The feature has also been proved by the studies in Australia [13] and Portugal [18].

\begin{tabular}{|l|l|l|}
\hline Age & Male & Female \\
\hline 18 & 0.32 & 0.47 \\
\hline 28 & 0.38 & 0.41 \\
\hline 38 & 0.50 & 0.52 \\
\hline 48 & 0.50 & 0.58 \\
\hline
\end{tabular}

Table 3: Pearson's correlation between third molar and age $(\mathrm{P}<0.01)$.

\begin{tabular}{|l|l|l|l|l|}
\hline & 18 & 28 & 38 & 48 \\
\hline Mann-Whitney & 17226.50 & 17633.50 & 22038.00 & 22496.00 \\
\hline
\end{tabular}

\begin{tabular}{|l|l|l|l|l|}
\hline$U$ & & & & \\
\hline$P$ & 0.86 & 0.50 & 0.26 & 0.57 \\
\hline
\end{tabular}

Table 4: Mann-Whitney U test between male and female.

\begin{tabular}{|c|c|c|c|c|c|c|}
\hline & Pearson & elation ${ }^{* *}$ & Wilcos & Test & & \\
\hline & & & Femal & & Male & \\
\hline & Female & Male & Z & Sample & $\mathbf{Z}$ & Sample \\
\hline $\begin{array}{l}18 \text { and } \\
28\end{array}$ & 0.90 & 0.91 & $-0.46^{*}$ & 236 & $1.51^{*}$ & 130 \\
\hline $\begin{array}{ll}38 & \text { and } \\
48 & \end{array}$ & 0.92 & 0.92 & $1.02^{*}$ & 290 & $-0.42^{*}$ & 154 \\
\hline $\begin{array}{l}18 \text { and } \\
48\end{array}$ & 0.57 & 0.53 & $6.17^{* *}$ & 248 & $4.27^{* *}$ & 130 \\
\hline $\begin{array}{ll}28 & \text { and } \\
38 & \end{array}$ & 0.51 & 0.57 & $4.84^{* *}$ & 243 & $5.05^{* *}$ & 139 \\
\hline
\end{tabular}

Table 5: The relationship between third molars.

\begin{tabular}{|c|c|c|c|c|c|c|c|c|}
\hline \multirow[b]{2}{*}{ Stage } & \multirow[b]{2}{*}{ Molars } & \multirow[b]{2}{*}{ Sample } & \multirow[b]{2}{*}{$\bar{x} \pm S$} & \multirow[b]{2}{*}{ Min } & \multirow[b]{2}{*}{ Max } & \multicolumn{3}{|c|}{ Percentiles } \\
\hline & & & & & & $25 \%$ & $50 \%$ & $75 \%$ \\
\hline \multirow[t]{3}{*}{ A } & 38 & 1 & $12.00 \pm-$ & 12.00 & 12.00 & 12.00 & 12.00 & 12.00 \\
\hline & 48 & 2 & $13.02 \pm 1.44$ & 12.00 & 14.03 & 12.00 & 13.02 & - \\
\hline & 18 & 11 & $12.99 \pm 1.13$ & 11.00 & 15.60 & 12.58 & 12.90 & 13.39 \\
\hline \multirow[t]{4}{*}{ B } & 28 & 7 & $12.82 \pm 1.03$ & 11.00 & 13.86 & 12.09 & 12.99 & 13.82 \\
\hline & 38 & 5 & $13.19 \pm 1.41$ & 11.08 & 14.47 & 11.85 & 13.39 & 14.44 \\
\hline & 48 & 8 & $13.20 \pm 1.10$ & 11.08 & 14.47 & 12.66 & 13.19 & 14.26 \\
\hline & 18 & 6 & $12.56 \pm 1.16$ & 11.00 & 14.23 & 11.46 & 12.62 & 13.53 \\
\hline \multirow[t]{4}{*}{ C } & 28 & 17 & $12.81 \pm 0.96$ & 11.00 & 14.32 & 12.35 & 12.90 & 13.51 \\
\hline & 38 & 8 & $12.55 \pm 1.31$ & 11.00 & 15.28 & 11.56 & 12.58 & 12.94 \\
\hline & 48 & 7 & $12.41 \pm 1.42$ & 11.00 & 15.28 & 11.54 & 12.09 & 12.99 \\
\hline & 18 & 113 & $13.95 \pm 1.42$ & 11.00 & 17.70 & 13.00 & 13.94 & 14.93 \\
\hline \multirow[t]{4}{*}{ D } & 28 & 114 & $13.99 \pm 1.45$ & 11.00 & 17.38 & 12.94 & 13.90 & 15.09 \\
\hline & 38 & 106 & $13.54 \pm 1.29$ & 11.00 & 17.70 & 12.77 & 13.41 & 14.33 \\
\hline & 48 & 101 & $13.58 \pm 1.34$ & 11.00 & 17.70 & 12.76 & 13.41 & 14.35 \\
\hline & 28 & 2 & $15.02 \pm 0.83$ & 14.43 & 15.61 & 14.43 & 15.02 & - \\
\hline \multirow[t]{2}{*}{$\mathrm{E}$} & 38 & 22 & $15.09 \pm 1.40$ & 12.00 & 17.26 & 14.19 & 15.28 & 16.18 \\
\hline & 48 & 23 & $14.86 \pm 1.41$ & 12.00 & 17.26 & 14.03 & 14.98 & 15.67 \\
\hline \multirow[t]{2}{*}{$\mathrm{F}$} & 38 & 5 & $15.23 \pm 0.57$ & 14.43 & 15.88 & 14.68 & 15.34 & 15.72 \\
\hline & 48 & 5 & $15.23 \pm 0.57$ & 14.43 & 15.88 & 14.68 & 15.34 & 15.72 \\
\hline
\end{tabular}


Citation: Ge-fei Shi, Xiao-peng Li, Rui-jue Liu, Jiang Tao3, Guang-you Zhu (2014) The Explore of Third Molar for Chronological Age Estimation in Teenagers of Shanghai Han Population. J Forensic Res 6: 1000266. doi:10.4172/2157-7145.1000266

Page 4 of 7

\begin{tabular}{|l|l|l|l|l|l|l|l|l|}
\hline & 18 & 3 & $15.34 \pm 2.31$ & 12.86 & 17.42 & 12.86 & 15.74 & - \\
\hline G & 28 & 3 & $15.34 \pm 2.31$ & 12.86 & 17.42 & 12.86 & 15.74 & - \\
\hline & 38 & 7 & $16.15 \pm 1.81$ & 12.86 & 18.62 & 15.54 & 16.13 & 17.42 \\
\hline & 48 & 7 & $16.15 \pm 1.81$ & 12.86 & 18.62 & 15.54 & 16.13 & 17.42 \\
\hline & 18 & 1 & $18.62 \pm-$ & 18.62 & 18.62 & 18.62 & 18.62 & 18.62 \\
\hline & 28 & 1 & $18.62 \pm-$ & 18.62 & 18.62 & 18.62 & 18.62 & 18.62 \\
\hline & 38 & 2 & $16.56 \pm 1.16$ & 15.74 & 17.38 & 15.74 & 16.56 & - \\
\hline & 48 & 2 & $16.56 \pm 1.16$ & 15.74 & 17.38 & 15.74 & 16.56 & - \\
\hline
\end{tabular}

Table 6: The age distribution as per stages of Demirjian's (Male).

\begin{tabular}{|c|c|c|c|c|c|c|c|c|}
\hline \multirow[b]{2}{*}{ Stage } & \multirow[b]{2}{*}{ Molars } & \multirow[b]{2}{*}{ Sample } & \multirow[b]{2}{*}{$\bar{x} \pm S$} & \multirow[b]{2}{*}{ Min } & \multirow[b]{2}{*}{ Max } & \multicolumn{3}{|c|}{ Percentiles } \\
\hline & & & & & & $25 \%$ & $50 \%$ & $75 \%$ \\
\hline & 18 & 2 & $12.45 \pm 0.45$ & 12.13 & 12.76 & 12.13 & 12.45 & - \\
\hline \multirow[t]{4}{*}{ A } & 28 & 1 & $12.13 \pm-$ & 12.13 & 12.13 & 12.13 & 12.13 & 12.13 \\
\hline & 38 & 6 & $12.53 \pm 0.81$ & 11.16 & 13.58 & 11.99 & 12.62 & 13.08 \\
\hline & 48 & 3 & $13.16 \pm 0.74$ & 12.49 & 13.95 & 12.49 & 13.04 & - \\
\hline & 18 & 11 & $12.39 \pm 1.26$ & 11.00 & 15.24 & 11.00 & 12.23 & 13.01 \\
\hline \multirow[t]{4}{*}{ B } & 28 & 9 & $13.07 \pm 2.17$ & 11.00 & 18.18 & 11.72 & 12.43 & 13.76 \\
\hline & 38 & 12 & $13.13 \pm 1.19$ & 11.00 & 15.26 & 12.14 & 13.34 & 13.97 \\
\hline & 48 & 13 & $12.49 \pm 1.08$ & 11.00 & 14.34 & 11.50 & 12.58 & 13.40 \\
\hline & 18 & 23 & $12.58 \pm 0.80$ & 11.00 & 14.26 & 12.10 & 12.50 & 13.00 \\
\hline \multirow[t]{4}{*}{ C } & 28 & 26 & $12.36 \pm 0.86$ & 11.00 & 13.75 & 11.47 & 12.61 & 13.00 \\
\hline & 38 & 11 & $12.60 \pm 1.39$ & 11.00 & 15.79 & 11.11 & 12.71 & 13.07 \\
\hline & 48 & 9 & $12.67 \pm 0.80$ & 11.11 & 13.73 & 12.19 & 12.71 & 13.33 \\
\hline & 18 & 211 & $13.94 \pm 1.71$ & 11.00 & 19.55 & 12.68 & 13.64 & 14.98 \\
\hline \multirow[t]{3}{*}{ D } & 28 & 206 & $13.96 \pm 1.73$ & 11.00 & 19.55 & 12.73 & 13.64 & 14.92 \\
\hline & 38 & 217 & $13.43 \pm 1.48$ & 11.00 & 18.26 & 12.41 & 13.22 & 14.10 \\
\hline & 48 & 217 & $13.40 \pm 1.43$ & 11.00 & 18.26 & 12.41 & 13.21 & 14.09 \\
\hline \multirow[t]{3}{*}{$\mathrm{E}$} & 38 & 21 & $15.27 \pm 1.41$ & 12.63 & 17.50 & 14.34 & 15.52 & 16.40 \\
\hline & 48 & 26 & $15.41 \pm 1.48$ & 12.63 & 17.62 & 14.27 & 15.53 & 16.57 \\
\hline & 18 & 1 & $12.85 \pm-$ & 12.85 & 12.85 & 12.85 & 12.85 & 12.85 \\
\hline \multirow[t]{4}{*}{$\mathrm{F}$} & 28 & 2 & $14.93 \pm 2.93$ & 12.85 & 17.00 & 12.85 & 14.93 & - \\
\hline & 38 & 17 & $15.44 \pm 1.63$ & 12.85 & 18.71 & 13.85 & 15.62 & 16.67 \\
\hline & 48 & 17 & $15.49 \pm 1.90$ & 12.85 & 19.01 & 13.72 & 15.53 & 16.89 \\
\hline & 18 & 5 & $17.48 \pm 1.68$ & 15.54 & 19.36 & 15.71 & 18.22 & 18.89 \\
\hline
\end{tabular}


Citation: Ge-fei Shi, Xiao-peng Li, Rui-jue Liu, Jiang Tao3, Guang-you Zhu (2014) The Explore of Third Molar for Chronological Age Estimation in Teenagers of Shanghai Han Population. J Forensic Res 6: 1000266. doi:10.4172/2157-7145.1000266

Page 5 of 7

\begin{tabular}{|l|l|l|l|l|l|l|l|l|}
\hline G & 28 & 5 & $16.32 \pm 2.02$ & 13.57 & 18.42 & 14.56 & 15.87 & 18.32 \\
\hline & 38 & 12 & $17.75 \pm 1.51$ & 15.54 & 19.55 & 16.15 & 17.98 & 19.13 \\
\hline & 48 & 11 & $17.63 \pm 1.53$ & 15.54 & 19.55 & 16.12 & 17.73 & 19.16 \\
\hline $\mathrm{H}$ & 18 & 4 & $18.35 \pm 1.20$ & 17.00 & 19.63 & 17.18 & 18.38 & 19.48 \\
\hline & 28 & 3 & $18.80 \pm 0.97$ & 17.73 & 19.63 & 17.73 & 19.03 & - \\
\hline & 38 & 2 & $19.03 \pm 0.86$ & 18.42 & 19.63 & 18.42 & 19.03 & - \\
\hline "Male and Female T-test of average age, $P>0.05$. & & $19.03 \pm 0.86$ & 18.42 & 19.63 & 18.42 & 19.03 & - \\
\hline
\end{tabular}

Table 7: The age distribution as per stages of demirjian's (female).

\begin{tabular}{|c|c|c|c|c|c|c|c|c|c|c|c|c|c|c|}
\hline \multirow{2}{*}{$\begin{array}{l}\text { Stage } \\
A\end{array}$} & \multirow{2}{*}{$\begin{array}{l}\text { Molars } \\
38\end{array}$} & \multirow{2}{*}{$\begin{array}{l}\text { Sample } \\
1\end{array}$} & \multicolumn{2}{|l|}{$\leq 14$} & \multicolumn{2}{|l|}{$\geq 14$} & \multicolumn{2}{|l|}{$\leq 16$} & \multicolumn{2}{|l|}{$\geq 16$} & \multicolumn{2}{|l|}{$\leq 18$} & \multicolumn{2}{|l|}{$\geq 18$} \\
\hline & & & $100.00 \%$ & (1) & - & $(-)$ & $100.00 \%$ & (1) & - & $(-)$ & $100.00 \%$ & (1) & - & $(-)$ \\
\hline & 48 & 2 & $50.00 \%$ & (1) & $50.00 \%$ & (1) & $100.00 \%$ & (2) & - & $(-)$ & $100.00 \%$ & (2) & - & $(-)$ \\
\hline & 18 & 11 & $90.91 \%$ & (10) & $9.09 \%$ & (1) & $100.00 \%$ & (11) & - & $(-)$ & $100.00 \%$ & (11) & - & $(-)$ \\
\hline \multirow[t]{4}{*}{ B } & 28 & 7 & $100.00 \%$ & (7) & - & $(-)$ & $100.00 \%$ & (7) & - & $(-)$ & $100.00 \%$ & (7) & - & $(-)$ \\
\hline & 38 & 5 & $60.00 \%$ & (3) & $40.00 \%$ & (2) & $100.00 \%$ & (5) & - & $(-)$ & $100.00 \%$ & (5) & - & $(-)$ \\
\hline & 48 & 8 & $75.00 \%$ & (6) & $25.00 \%$ & (2) & $100.00 \%$ & (8) & - & $(-)$ & $100.00 \%$ & (8) & - & $(-)$ \\
\hline & 18 & 6 & $83.33 \%$ & (5) & $16.67 \%$ & (1) & $100.00 \%$ & (6) & - & $(-)$ & $100.00 \%$ & (6) & - & $(-)$ \\
\hline \multirow[t]{4}{*}{ C } & 28 & 17 & $88.24 \%$ & (15) & $11.76 \%$ & (2) & $100.00 \%$ & (17) & - & $(-)$ & $100.00 \%$ & (17) & - & $(-)$ \\
\hline & 38 & 8 & $87.50 \%$ & (7) & $12.50 \%$ & (1) & $100.00 \%$ & (8) & - & $(-)$ & $100.00 \%$ & (8) & - & $(-)$ \\
\hline & 48 & 7 & $85.71 \%$ & (6) & $14.29 \%$ & (1) & $100.00 \%$ & (7) & - & $(-)$ & $100.00 \%$ & (7) & - & $(-)$ \\
\hline & 18 & 113 & $53.10 \%$ & (60) & $47.79 \%$ & (54) & $92.92 \%$ & (105) & $7.08 \%$ & (8) & $100.00 \%$ & (113) & - & $(-)$ \\
\hline \multirow[t]{4}{*}{ D } & 28 & 114 & $51.75 \%$ & (59) & $49.12 \%$ & (56) & $92.11 \%$ & (105) & $7.89 \%$ & (9) & $100.00 \%$ & (114) & - & $(-)$ \\
\hline & 38 & 106 & $68.87 \%$ & (73) & $32.08 \%$ & (34) & $97.17 \%$ & (103) & $2.83 \%$ & (3) & $100.00 \%$ & (106) & - & $(-)$ \\
\hline & 48 & 101 & $69.31 \%$ & (70) & $31.68 \%$ & (32) & $95.05 \%$ & (96) & $4.95 \%$ & (5) & $100.00 \%$ & (101) & - & $(-)$ \\
\hline & 28 & 2 & - & $(-)$ & $100.00 \%$ & (2) & $100.00 \%$ & (2) & - & $(-)$ & $100.00 \%$ & (2) & - & $(-)$ \\
\hline \multirow[t]{2}{*}{$E$} & 38 & 22 & $18.18 \%$ & (4) & $81.82 \%$ & (18) & $68.18 \%$ & (15) & $31.82 \%$ & (7) & $100.00 \%$ & (22) & - & $(-)$ \\
\hline & 48 & 23 & $21.74 \%$ & (5) & $78.26 \%$ & (18) & $78.26 \%$ & (18) & $21.74 \%$ & (5) & $100.00 \%$ & (23) & - & $(-)$ \\
\hline \multirow[t]{3}{*}{$\mathrm{F}$} & 38 & 5 & - & $(-)$ & $100.00 \%$ & (5) & $100.00 \%$ & (5) & - & $(-)$ & $100.00 \%$ & (5) & - & $(-)$ \\
\hline & 48 & 5 & - & $(-)$ & $100.00 \%$ & (5) & $100.00 \%$ & (5) & - & $(-)$ & $100.00 \%$ & (5) & - & $(-)$ \\
\hline & 18 & 3 & $33.33 \%$ & (1) & $66.67 \%$ & (2) & $66.67 \%$ & (2) & $33.33 \%$ & (1) & $100.00 \%$ & (3) & - & $(-)$ \\
\hline \multirow[t]{4}{*}{ G } & 28 & 3 & $33.33 \%$ & (1) & $66.67 \%$ & (2) & $66.67 \%$ & (2) & $33.33 \%$ & (1) & $100.00 \%$ & (3) & - & $(-)$ \\
\hline & 38 & 7 & $14.29 \%$ & (1) & $85.71 \%$ & (6) & $42.86 \%$ & (3) & $57.14 \%$ & (4) & $85.71 \%$ & (6) & $14.29 \%$ & (1) \\
\hline & 48 & 7 & $14.29 \%$ & (1) & $85.71 \%$ & (6) & $42.86 \%$ & (3) & $57.14 \%$ & (4) & $85.71 \%$ & (6) & $14.29 \%$ & (1) \\
\hline & 18 & 1 & - & $(-)$ & $100.00 \%$ & (1) & - & $(-)$ & $100.00 \%$ & (1) & - & $(-)$ & $100.00 \%$ & (1) \\
\hline $\mathrm{H}$ & 28 & 1 & - & $(-)$ & $100.00 \%$ & (1) & - & $(-)$ & $100.00 \%$ & (1) & - & $(-)$ & $100.00 \%$ & (1) \\
\hline
\end{tabular}


Citation: Ge-fei Shi, Xiao-peng Li, Rui-jue Liu, Jiang Tao3, Guang-you Zhu (2014) The Explore of Third Molar for Chronological Age Estimation in Teenagers of Shanghai Han Population. J Forensic Res 6: 1000266. doi:10.4172/2157-7145.1000266

Page 6 of 7

\begin{tabular}{|l|l|l|l|l|l|l|l|l|l|l|l|l|l|l|}
\hline & 38 & 2 & - & $(-)$ & $100.00 \%$ & $(2)$ & $50.00 \%$ & $(1)$ & $50.00 \%$ & $(1)$ & $100.00 \%$ & $(2)$ & - & $(-)$ \\
\hline & 48 & 2 & - & $(-)$ & $100.00 \%$ & $(2)$ & $50.00 \%$ & $(1)$ & $50.00 \%$ & $(1)$ & $100.00 \%$ & $(2)$ & - & $(-)$ \\
\hline
\end{tabular}

Table 8: The probability of estimating 14-year, 16-year and 18-year by third molars (Male).

\begin{tabular}{|c|c|c|c|c|c|c|c|c|c|c|c|c|c|c|}
\hline \multirow{2}{*}{$\begin{array}{l}\text { Stage } \\
\text { A }\end{array}$} & \multirow{2}{*}{$\begin{array}{l}\text { Molars } \\
18\end{array}$} & \multirow{2}{*}{$\begin{array}{l}\text { Sample } \\
2\end{array}$} & \multicolumn{2}{|l|}{$\leq 14$} & \multicolumn{2}{|l|}{$\geq 14$} & \multicolumn{2}{|l|}{$\leq 16$} & \multicolumn{2}{|l|}{$\geq 16$} & \multicolumn{2}{|l|}{$\leq 18$} & \multicolumn{2}{|l|}{$\geq 18$} \\
\hline & & & $100.00 \%$ & (2) & - & $(-)$ & $100.00 \%$ & (2) & - & $(-)$ & $100.00 \%$ & (2) & - & $(-)$ \\
\hline & 28 & 1 & $100.00 \%$ & (1) & - & $(-)$ & $100.00 \%$ & (1) & - & $(-)$ & $100.00 \%$ & (1) & - & $(-)$ \\
\hline & 38 & 6 & $100.00 \%$ & (6) & - & $(-)$ & $100.00 \%$ & (6) & - & $(-)$ & $100.00 \%$ & (6) & - & $(-)$ \\
\hline & 48 & 3 & $100.00 \%$ & (3) & - & $(-)$ & $100.00 \%$ & (3) & - & $(-)$ & $100.00 \%$ & (3) & - & $(-)$ \\
\hline \multirow[t]{4}{*}{$B$} & 18 & 11 & $90.91 \%$ & (10) & $9.09 \%$ & (1) & $100.00 \%$ & (11) & - & $(-)$ & $100.00 \%$ & (11) & - & $(-)$ \\
\hline & 28 & 9 & $77.78 \%$ & (7) & $22.22 \%$ & (2) & $88.89 \%$ & (8) & $11.11 \%$ & (1) & $88.89 \%$ & (8) & $11.11 \%$ & (1) \\
\hline & 38 & 12 & $75.00 \%$ & (9) & $25.00 \%$ & (3) & $100.00 \%$ & (12) & - & $(-)$ & $100.00 \%$ & (12) & - & $(-)$ \\
\hline & 48 & 13 & $92.31 \%$ & (12) & $7.69 \%$ & (1) & $100.00 \%$ & (13) & - & $(-)$ & $100.00 \%$ & (13) & - & $(-)$ \\
\hline \multirow[t]{4}{*}{ C } & 18 & 23 & $95.65 \%$ & (22) & $4.35 \%$ & (1) & $100.00 \%$ & (23) & - & $(-)$ & $100.00 \%$ & (23) & - & $(-)$ \\
\hline & 28 & 26 & $100.00 \%$ & (26) & - & $(-)$ & $100.00 \%$ & (26) & - & $(-)$ & $100.00 \%$ & (26) & - & $(-)$ \\
\hline & 38 & 11 & $90.91 \%$ & (10) & $9.09 \%$ & (1) & $100.00 \%$ & (11) & - & $(-)$ & $100.00 \%$ & (11) & - & $(-)$ \\
\hline & 48 & 9 & $100.00 \%$ & (9) & - & $(-)$ & $100.00 \%$ & (9) & - & $(-)$ & $100.00 \%$ & (9) & - & $(-)$ \\
\hline \multirow[t]{4}{*}{ D } & 18 & 211 & $60.66 \%$ & (128) & $40.76 \%$ & (86) & $87.20 \%$ & (184) & $12.80 \%$ & (27) & $97.63 \%$ & (206) & $2.37 \%$ & (5) \\
\hline & 28 & 206 & $61.65 \%$ & (127) & $39.81 \%$ & (82) & $87.38 \%$ & (180) & $12.62 \%$ & (26) & $96.60 \%$ & (199) & $3.40 \%$ & (7) \\
\hline & 38 & 217 & $71.89 \%$ & (156) & $29.49 \%$ & (64) & $93.55 \%$ & (203) & $6.45 \%$ & (14) & $98.62 \%$ & (214) & $1.38 \%$ & (3) \\
\hline & 48 & 217 & $72.81 \%$ & (158) & $28.57 \%$ & (64) & $94.47 \%$ & (205) & $5.53 \%$ & (12) & $98.62 \%$ & (214) & $1.38 \%$ & (3) \\
\hline \multirow[t]{2}{*}{ E } & 38 & 21 & $23.81 \%$ & (5) & $76.19 \%$ & (16) & $66.67 \%$ & (14) & $33.33 \%$ & (7) & $100.00 \%$ & (21) & - & $(-)$ \\
\hline & 48 & 26 & $19.23 \%$ & (5) & $80.77 \%$ & (21) & $61.54 \%$ & (16) & $38.46 \%$ & (10) & $100.00 \%$ & (26) & - & $(-)$ \\
\hline \multirow[t]{4}{*}{$\mathrm{F}$} & 18 & 1 & $100.00 \%$ & (1) & - & $(-)$ & $100.00 \%$ & (1) & - & $(-)$ & $100.00 \%$ & (1) & - & $(-)$ \\
\hline & 28 & 2 & $50.00 \%$ & (1) & $50.00 \%$ & (1) & $50.00 \%$ & (1) & $50.00 \%$ & (1) & $100.00 \%$ & (2) & - & $(-)$ \\
\hline & 38 & 17 & $29.41 \%$ & (5) & $70.59 \%$ & (12) & $58.82 \%$ & (10) & $41.18 \%$ & (7) & $94.12 \%$ & (16) & $5.88 \%$ & (1) \\
\hline & 48 & 17 & $35.29 \%$ & (6) & $64.71 \%$ & (11) & $58.82 \%$ & (10) & $41.18 \%$ & (7) & $88.24 \%$ & (15) & $11.76 \%$ & (2) \\
\hline \multirow[t]{4}{*}{ G } & 18 & 5 & - & $(-)$ & $100.00 \%$ & (5) & $40.00 \%$ & (2) & $60.00 \%$ & (3) & $40.00 \%$ & (2) & $60.00 \%$ & (3) \\
\hline & 28 & 5 & $20.00 \%$ & (1) & $80.00 \%$ & (4) & $60.00 \%$ & (3) & $40.00 \%$ & (2) & $60.00 \%$ & (3) & $40.00 \%$ & (2) \\
\hline & 38 & 12 & - & $(-)$ & $100.00 \%$ & (12) & $16.67 \%$ & (3) & $83.33 \%$ & (10) & $50.00 \%$ & (6) & $50.00 \%$ & (6) \\
\hline & 48 & 11 & - & $(-)$ & $100.00 \%$ & (11) & $18.18 \%$ & (2) & $81.82 \%$ & (9) & $54.55 \%$ & (6) & $45.45 \%$ & (5) \\
\hline \multirow[t]{3}{*}{$\mathrm{H}$} & 18 & 4 & - & $(-)$ & $100.00 \%$ & (4) & - & $(-)$ & $100.00 \%$ & (4) & $50.00 \%$ & (2) & $50.00 \%$ & (2) \\
\hline & 28 & 3 & - & $(-)$ & $100.00 \%$ & (3) & - & $(-)$ & $100.00 \%$ & (3) & $33.33 \%$ & (1) & $66.67 \%$ & (2) \\
\hline & 38 & 2 & - & $(-)$ & $100.00 \%$ & (2) & - & $(-)$ & $100.00 \%$ & (2) & - & $(-)$ & $100.00 \%$ & (2) \\
\hline
\end{tabular}


48 2

$-$

$(-)$
$100.00 \%$

(2)

$-$

$(-)$

\begin{tabular}{|l|l|l}
\hline $100.00 \%$ & $(2)$ & -
\end{tabular}

$(-)$

$100.00 \% \quad(2)$

Table 9: The probability of estimating 14-year, 16-year and 18-year by third molars (Female).

It is reported that $99.5 \%$ (male) and $99.3 \%$ (female) Australian youngster is at least 18-year age when third molar mineralization reaches stage $\mathrm{H}$ [13]. Our findings are: When 18, 28, 38 and 48 is classified as stage $\mathrm{H}$ respectively, $50 \%, 66.7 \%, 100 \%$ and $100 \%$ Shanghai female and $100 \%, 100 \%, 100 \%$ and $100 \%$ Shanghai male are beyond 18-year-old. When 38,48 are classified as stage G, $83.3 \%$ and $81.8 \%$ female are beyond 16 -year-old and $50 \%$ and $54.5 \%$ female are below 18 -year-old. Meanwhile, $57.1 \%$ and $57.1 \%$ male are beyond $16-$ year-old and $85.7 \%$ and $85.7 \%$ male are below 18 -year-old. When 18 , 28,38 and 48 are classified as stage D, 87.2\%, 87.4\%, 93.6\%, 94.5\% female and $92.9 \%, 92.1 \%, 97.2 \%, 95.1 \%$ male are below 16-year-old respectively. When $18,28,38$ and 48 are classified as stage C, $95.7 \%$, $100 \%, 90.9 \%, 100 \%$ female and $83.3 \%, 88.2 \%, 87.5 \%$ and $85.7 \%$ male are below 14-year-old respectively. This study, however, indicates that, stage $\mathrm{C}, \mathrm{D}$ and $\mathrm{G}$ is respectively landmark for judging less than 14 years old, 16 years old and 18 years old.

The study shows that the difference between the groups, and difference from other studies, may be closely related to the sample specificity. What Olze et al. [19] studied has shown this before. Although this view has not been further confirmed, but was really found by some going researches. However, some expert holds that there is no evidence of difference among countries in third molar development [20]. Based on these, we do not recommend that the results are used to other population.

\section{Conclusion}

Our study concludes that there is no difference between female and male in third molar development, and between both sides. The significant difference, although, is found between 18 and 48, 28 and 38 .

Stage C, D and G is respectively landmark for judging less than 14year-old, 16-year-old and 18-year-old. As a conclusion, third molar could be used to estimate the age of Shanghai teenagers. But there is different accuracy rate for the different classification of the third molar. The further study will be done.

\section{References}

1. Yan J, Lou X, Xie L, Yu D, Shen G (2013) Assessment of Dental Age of Children Aged 3.5 to 16.9 Years Using Demirjian's Method: A MetaAnalysis Based on 26 Studies. PLoS ONE 8: e84672.

2. Andreas Olze, Dominique Bilang, Sven Schmidt, Klaus-Dieter Wernecke, Gunther Geserick, et al., (2004) Forensic age estimation in living subjects the ethnic factor in wisdom tooth mineralization. Int J Legal Med 118: 170-173.

3. Andreas Olze, Dominique Bilang, Sven Schmidt, Klaus-Dieter Wernecke, Gunther Geserick, et al., (2005) Validation of common classification systems for assessing the mineralization of third molars. Int J Legal Med 119: 22-26.

4. Orhana K, Ozerb, Orhanb AI, Doganb S, Paksoya CS (2007) Radiographic evaluation of third molar development in relation to chronological age among Turkish children and youth. Forensic Sci Int 165: 46-51.

5. Soo-Hyun Lee, Jeong-Yun Lee, Hee-Kyung Park, Young-Ku Kim (2009) Development of third molars in Korean juveniles and adolescents. Forensic Science International 188: 107-111.

6. Cruz-Landeira A, Linares-Argote J, Rodríguez MM, Rodríguez-Calvo MS, Otero XL (2010) Dental age estimation in Spanish and Venezuelan children. Comparison of Demirjian and Chaillet's scores. Int J Legal Med 124: 105-112.

7. Loredana Golovcencu, Călin Scripcaru, Georgeta Zegan (2007) Thirdmolar development in relation to chronologic age in Turkish children and young adults. Angle Orthod 77: 1040-1045.

8. José LP, Elena B, Ricardo O, Concepción M (2005) Evaluation of chronological age based on third molar development in the Spanish population. Int J Legal Med 119: 349-354.

9. Arany SM, Iino, Yoshioka N (2004) Radiographic survey of third molar development in relation to chronological age among Japanese juveniles. J Forensic Sci 49: 534-538.

10. Mincer HH, Harris EF, Berryman HE (1993) The A.B.F.O. study of third molar development and its use as an estimator of chronological age. J Forensic Sci 38: 379-90.

11. Levesque GY, Demirjian A, Tanguay R (1981) Sexual Dimorphism in the Development, Emergence, and Agenesis of the Mandibular Third Molar. Journal of Dental Research 60: 1735-1741.

12. Gunst K, Mesotten K, Carbonez K, Willems G (2003) Third molar root development in relation to chronological age: a large sample sized retrospective study. Forensic Sci Int 136: 52-57.

13. Meinla A, Tanglc S, Hubera s, Maurerd B, Watzekc G (2007) The chronology of third molar mineralization in the Austrian population-a contribution to forensic age estimation. Forensic Sci Int 169: 161-167.

14. Zeng DL, Wu ZL, Cui MY (2010) Chronological age estimation of third molar mineralization of Han in southern China. Int J Legal Med 124: 119-123.

15. Olzea A, Van Niekerkb P, Schmidta S, Werneckec D, Rösingd FW, Gesericka G et al., (2006) Studies on the progress of third-molar mineralisation in a Black African population. Homo 57: 209-217.

16. MaríaVictoria Bolañosa, Hasnae Moussab, María Cinta Manriquea, Manuel Jorge Bolañosc (2003) Radiographic evaluation of third molar development in Spanish children and young people. Forensic Sci Int 133: 212-219.

17. Olzea A, Ishikawab T, Zhub BL, Schulzc R, Heinecked A, et al., (2008) Studies of the chronological course of wisdom tooth eruption in a Japanese population. Forensic Sci Int 174: 203-206.

18. Inês Morais Caldas, Paula Júlio, Ricardo Jorge Simões, Eduarda Matos, Américo Afonso et al., (2011) Chronological age estimation based on third molar development in a Portuguese population. Int J Legal Med 125: 235-243.

19. Schmeling A, Reisinger W, Geserick G, Olze A (2006) Age estimation of unaccompanied minors. Part II. Dental aspects. Forensic Sci Int 159: 65-67.

20. Thevissen PW, Fieuws S, Willems G (2010) Human third molars development: Comparison of 9 country specific populations. Forensic Sci Int 201: 102-105. 